\title{
The determination of Polonium-210 in urine following the Litvinenko incident
}

\author{
G.J. Ham \\ Health Protection Agency, Centre for Radiation, Chemical and Environmental Hazards, \\ OX11 ORQ Chilton, Didcot, Oxon, UK
}

\begin{abstract}
Following the confirmation that Mr Litvinenko had received a large dose of ${ }^{210} \mathrm{Po}$, the Health Protection Agency began to prepare for public health monitoring of potentially contaminated people. Alpha counting of urine was the only practicable means of monitoring exposure. As the incident developed it became apparent that the number of samples would be likely to exceed the HPA's capacity. Therefore other UK laboratories were contacted and several agreed to assist. To validate the analyses each potential collaborator analysed a cross check sample that was the remainder from 24 hour samples that had already been measured at the HPA. The results were in good agreement. In the first four weeks of the programme, about 600 samples were collected and all were analysed using the resources in the UK. Later, the quality assurance exercise was extended to include laboratories in the UK and abroad that could potentially become involved in the analysis of samples arising from the incident. The factors underlying the success of the programme are set out. These included having a well founded Quality Assurance system in place during normal circumstances and having pre-existing links at the appropriate working level with other laboratories in the UK and abroad.
\end{abstract}

\section{INTRODUCTION}

A number of buildings and vehicles in London became contaminated with polonium-210 $\left({ }^{210} \mathrm{Po}\right)$ in connection with the poisoning of Mr. Alexander Litvinenko. Some staff at these premises, guests at the hotels, visitors to the offices, and other members of the general public were also potentially contaminated. In order to determine the extent of any contamination, samples of urine have to date been taken from over 700 people. Each individual is asked to collect all of the urine produced over a $24 \mathrm{~h}$ period. This paper describes how the programme of sampling and analysis was set up and executed by the Health Protection Agency (HPA). The methods used to calculate the committed effective doses to the individuals who provided urine samples have been described elsewhere. Monitoring of buildings etc for contamination was performed by HPA but this work is not described here. Summaries of such work have been published on the HPA web site [1].

\section{MONITORING DESIGN}

\subsection{Initial assessment of the situation}

In late November 2006 HPA was informed that Mr. Litvinenko, whose illness was widely reported in the press, had a significant quantity of ${ }^{210} \mathrm{Po}$ in his body. A team of experts in the Radiation Protection Division (RPD) made an initial assessment of potential risks to those who had been in contact with him from the presumed time at which he was poisoned, i.e. the public health consequences, and collected information on natural levels of ${ }^{210}$ Po in human body tissues and excreta.

Since ${ }^{210} \mathrm{Po}$ is almost a pure alpha-emitter, there would be no external hazard from the victim's body. Normal hygiene and cleanliness practices in hospitals should have reduced the likelihood of any significant intake by NHS staff and others and therefore any radiation hazard. 


\subsection{Choice of monitoring}

The environmental (surface contamination) monitoring of places associated with the incident provided information on the remaining hazard, from which advice on the need for any remediation measures could be given. However, this approach gave only limited information on the original extent of contamination, because of activities such as cleaning that had taken place in the intervening period.

In order to confirm that doses from secondary contamination from the victim (and perhaps other persons) were low, and to determine any exposures resulting from inadvertent exposure to the source material, consideration was given to the possibilities for individual monitoring for ${ }^{210} \mathrm{Po}$. This would enable any exposures that had occurred to be assessed directly and allow the resultant individual risks to be determined. Because of the low level of photon emissions from ${ }^{210} \mathrm{Po}$, it is not possible to determine the amount of activity in the body using external radiation detectors. However, there is ongoing excretion of about $1.5 \%$ per day of the systemic activity, so consideration was given to measurements in excreta.

In the case of ${ }^{210} \mathrm{Po}$, measurements using faeces have two advantages over those using urine. Faeces are the predominant excretion route and so there would be more activity present in the sample and the sensitivity of the measurements would be greater. However, faecal monitoring has several disadvantages. There is greater day-to-day variation in the amount of faeces excreted than is the case for urine. In some workplaces, faecal samples are collected over a 3-day period to take account of this. There were obvious practical problems in the collection and containment of faecal samples, particularly since the provision of samples by members of the public was going to be voluntary. The analysis of faecal samples would also be more problematical than dealing with urine. Overall, urine was the material selected because of the likelihood that more people would choose to provide samples, and because of the fewer problems associated with sample handling and analysis. Urine sampling has been used extensively for monitoring of workers potentially exposed to ${ }^{210} \mathrm{Po}$, and practical experience in the analysis had been published [2]. Further considerations were that ${ }^{210} \mathrm{Po}$ is present in all of us, and the ambient levels in urine were expected to be detectable using established analytical methods.

\subsection{Natural levels of polonium-210 in urine}

Polonium-210 is present in environmental materials, including foodstuffs, drinking water and in the air. It is also formed within the body as a result of intakes of lead-210 $\left({ }^{210} \mathrm{~Pb}\right)$, which decays to ${ }^{210} \mathrm{Po}$. An initial search of the literature was carried to elicit measurements of ambient levels of ${ }^{210}$ Po in human urine, i.e., those from dietary and other intakes of ${ }^{210} \mathrm{~Pb}$ and ${ }^{210} \mathrm{Po}$ (e.g. cigarette smoke). This review also considered factors that could account for enhanced ambient levels such as unusually high intakes of particular foods, or time spent in areas of high natural background.

The results of this initial survey, carried out in the first day or so following the identification of ${ }^{210} \mathrm{Po}$ in the victim, are summarised in Table 1 . Most results in Table 1 fall in the range $5-15 \mathrm{mBq}$ per day, with higher levels within this range being found in smokers. It was therefore considered that results above $30 \mathrm{mBq}$ were very unlikely to be due entirely to ambient excretion.

Table 1. Reported values of ${ }^{210} \mathrm{Po}$ excretion in urine.

\begin{tabular}{|l|l|l|}
\hline Country & Subjects & Range \\
\hline USA [3] & & $9.3 \mathrm{mBq} \mathrm{d}^{-1}$ \\
\hline Germany [4] & & Detection limit $\left(2 \mathrm{mBq}^{-1 *}\right)$ to $9.9 \mathrm{mBq} \mathrm{d}^{-1}$ \\
\hline Brazil [5] & Non-smokers & $5.2+/-2.2 \mathrm{mBq} \mathrm{d}^{-1}$ \\
& Smokers & $9.9+/-4.1 \mathrm{mBq} \mathrm{d}^{-1}$ \\
\hline Saudi Arabia [6] & Non-smokers & 1.5 to $10 \mathrm{mBql}^{-1 *}$ \\
& Smokers & 3.3 to $15.9 \mathrm{mBql}^{-1 *}$ \\
& Shisa smokers & 2.2 to $19.6 \mathrm{mBql}^{-1 *}$ \\
\hline *Typical daily excretion of urine is $1.41 . \mathrm{d}^{-1}$ making a detection limit of $2 \mathrm{mBq}^{-1}$ equivalent to $3 \mathrm{mBq} \mathrm{d}^{-1}$ \\
\hline
\end{tabular}




\subsection{Criteria for analytical method}

The HPA measurements were designed for public health purposes. The primary aim was to assess any exposures to members of the public and various employees who may have had contact with a contaminated person or location. An excretion rate of $30 \mathrm{mBq} \mathrm{d}^{-1}$ in urine was therefore chosen as the "Reporting Level" (RL) above which a dose would be calculated and reported. This RL corresponds to a minimum detectable committed effective dose $\mathrm{E}(50)$ of about $0.3 \mathrm{mSv}$ (for a urine sample obtained 20 days after intake) based on the cautious assumption of $100 \%$ intake by inhalation. The fraction of the intake excreted in urine per day decreases with time after intake, as the activity remaining in the body decreases. Thus for a given daily excretion rate, the longer the delay between the intake of ${ }^{210}$ Po and the sample being collected the larger is the assessed dose. However, if a sample collected $100 \mathrm{~d}$ after the assumed intake gave an excretion rate of $30 \mathrm{mBq} \mathrm{d}^{-1}$, then the assessed dose would be about $1 \mathrm{mSv}$. It was therefore feasible to confirm that doses were well below levels considered to be of some concern, even for urine samples collected several months after intake.

Because of the expected public concern, the method chosen needed to produce analytical data as rapidly as possible and it was also anticipated that the first samples would arrive at the HPA's analytical laboratory within 2-4 days of the HPA becoming aware of the incident. It was therefore essential that the method could be used at very short notice, utilising existing equipment and supplies and with a minimum of operator training and validation.

\subsection{Method development}

Given the above criteria it was decided to adapt the method that was in routine operational use at the HPA laboratory for the analysis of environmental samples such as foodstuffs. This is summarised as follows:

1. Measure 1 litre of urine sample using a measuring cylinder into a 2-litre beaker.

2. Add ${ }^{209}$ Po or ${ }^{208}$ Po yield tracer (typically about $0.2 \mathrm{~Bq}$ )

3. Add $200 \mathrm{ml}$ of concentrated nitric acid. Heat on a hotplate set at $200{ }^{\circ} \mathrm{C}$ with occasional stirring. The sample should go straw coloured over time.

4. Evaporate sample to dryness (overnight on a hotplate set at $150{ }^{\circ} \mathrm{C}$ ).

5. Cover the residue with the minimum quantity of concentrated hydrochloric acid (enough to dissolve the residue with warming) and take to dryness (hotplate $200^{\circ} \mathrm{C}$ ). Repeat this step.

6. Dissolve the residue with $6 \mathrm{M}$ hydrochloric acid and transfer to a suitable beaker (250-600 $\mathrm{ml}$ tall form beaker) using $6 \mathrm{M}$ hydrochloric acid. Make up to half beaker volume using $6 \mathrm{M}$ hydrochloric acid.

7. Add $1 \mathrm{ml}$ of $30 \% \mathrm{w} / \mathrm{v}$ hydroxyl ammonium chloride solution and a magnetic stirrer. Adjust the $\mathrm{pH}$ of the solution with stirring to 2 , with 0.880 ammonia or hydrochloric acid.

8. Heat the solution with stirring to at least $85^{\circ} \mathrm{C}$. Add a polished silver disc contained within a disposable holder such that only one side of the disc is exposed to the solution. Spontaneously deposit the polonium on to the silver disc over a $3 \mathrm{~h}$ period.

9. Remove the disc from the holder, dry with ethanol and count overnight in a solid state alpha spectrometer. A counting time of 12 hours should be long enough to measure a minimum detectable activity (MDA) of $20 \mathrm{mBq}$ for a 24-hour sample.

Several one litre samples of urine were collected from staff and analysed for polonium using this scheme. Radiochemical yields were reasonable (better than $30 \%$ ) and ${ }^{210}$ Po concentrations equivalent to excretion rates of between 4 and $10 \mathrm{mBq}$ per day were measured. Radioanalytical results were available within 36-48 hours after receipt of sample. At that stage the capacity of the HPA's Chilton laboratories was estimated to be about 20 samples per day. 


\section{MONITORING STRATEGY}

It was apparent at the start of the incident that while the initial requirement was to monitor a relatively small number of people, there was a potential for the numbers of samples to increase rapidly to beyond the available analytical capacity. Because of this, the strategy adopted was that potential contacts would be interviewed to ascertain their potential for exposure. Those with the highest likelihood would then be selected for monitoring. The measurement results on these samples could then be used to re-evaluate any need for monitoring of others and reassure people with lower potential of exposure.

Despite this strategy it was not possible to estimate the likely number of samples. It was anticipated however that the HPA's analytical capacity could be exceeded. Six public sector research establishments, including HPA, had signed a co-operation agreement in 2005. As a precaution these laboratories and others in the UK known to be capable of measuring ${ }^{210}$ Po were contacted and asked if they would be able to provide assistance if required. Initial responses suggested that the total UK capacity would be about 60-100 samples a day. Two other laboratories, both part of the co-operation agreement, were capable of carrying out the necessary analyses and agreed to stand by in case they were needed. HPA made use of its radioanalytical facilities at Chilton and Glasgow.

\section{THE MONITORING PROCESS}

\subsection{Sampling and reporting of results}

The HPA's Local and Regional Services (LARS) developed the triage procedures to identify those with highest potential for exposure. After evaluating the questionnaire they requested urine samples from identified individuals and arranged the collection. Samples were then transported to the HPA's laboratory at Chilton where they were logged. The samples were then either analysed at Chilton or passed on to collaborating laboratories for analysis. The HPA's Radiological Protection Division then assessed doses and reported results to LARS who communicated them to individuals putting them in perspective. The availability of the staff in LARS who were experienced both in triage and in communicating with the general public was essential to the smooth operation of the process.

The triage questionnaire for health care staff was designed to determine the extent of contact with Mr. Litvinenko or his bodily fluids. The one for members of the public and hotel staff was designed to determine if people were in designated locations at specific times. The monitoring results from buildings determined the targeting of specific locations which were agreed with the other public bodies investigating the incident.

\subsection{Collaboration between laboratories}

Collaboration between laboratories was greatly aided both by the existence of the co-operation agreement and by the fact that the radioanalytical community is relatively small. Radioanalaytical staff at Chilton had already established contact with potential collaborating laboratories via scientific meetings and through intercomparison exercises. The use of a method which is in routine use by a laboratory's staff is more likely to achieve satisfactory results than using an unfamiliar one. It was therefore decided that any collaborating laboratories should use their existing analytical methods (suitably adapted for the purpose), rather than all adopt the one that was in use at the HPA.

With the agreement of the other laboratories it was decided that before any collaborating laboratory started work on samples, they would analyse an intercomparison sample. These samples were the remainder of actual samples that had been analysed at the HPA. This gave reassurance that the results from different laboratories were comparable with each other. Since different methods were being used, the comparability of data gave added confidence in the reliability of the results. Later, it was considered that the UK's analytical capacity could be overwhelmed and so other European laboratories agreed to 
stand by and also analysed intercomparison samples. The intercomparison was extended even further when laboratories that had analysed samples from their nationals who had been potentially contaminated in London requested samples.

The results of the intercomparison exercise were very good. All measurements were in agreement with each other. Results are summarised in Table 2.

Table 2. Results of intercomparison exercise.

\begin{tabular}{|c|c|c|c|c|c|c|c|}
\hline \multicolumn{8}{|c|}{ Measured ${ }^{210} \mathrm{Po}$ concentration in $\mathrm{mBq}^{-1}$} \\
\hline HPA & $23 \pm 7^{*}$ & $\mathbf{1 7} \pm \mathbf{6}^{*}$ & $44 \pm 12^{*}$ & $12 \pm 4$ & $197 \pm 30$ & $13 \pm 4$ & $193 \pm 23$ \\
\hline Other Lab & $\mathbf{2 9} \pm 13^{*}$ & $16 \pm \mathbf{3}^{*}$ & $46 \pm 12^{*}$ & $18 \pm 5$ & $174 \pm 10$ & $16 \pm 7$ & $146 \pm 9$ \\
\hline HPA & $60 \pm 10$ & $48 \pm 8$ & $242 \pm 32$ & $242 \pm 32$ & $76 \pm 15$ & $36 \pm 6$ & $45 \pm 8$ \\
\hline Other Lab & $80 \pm 8$ & $66 \pm 9$ & $195 \pm 11$ & $190 \pm 15$ & $58 \pm 5$ & $38 \pm 6$ & $58 \pm 12$ \\
\hline
\end{tabular}

\subsection{Quantity of samples processed}

As the incident response progressed the number of samples processed each day steadily increased to a peak of about 50. By the end of $2006(\approx 30$ days after samples had first been received) 548 samples had been analysed. The pace then slowed somewhat and during January 2007 about another 120 samples were processed. In total the incident required the analysis of over 700 urine samples. The increase in sample numbers was so rapid that the HPA had to send samples to collaborating laboratories at the end of the first week of sampling and the collaborating laboratories performed about $20 \%$ of the total analyses. Despite the rapid onset, no samples were held awaiting analysis at any time.

\section{CONCLUSIONS AND IMPLICATIONS FOR THE FUTURE}

The incident highlighted several issues to the HPA many of which are of general interest to those planning emergency response.

The presence of a pre-existing quality assurance scheme (in the HPA's case accredited to both ISO 9001 and 17025), meant that there was a well developed quality culture in the laboratory. Even though the modified method was outside the scope of our ISO17025 accreditation it was routine to use the same quality assurance procedures in this work. This minimised the possibility of errors and the results of the intercomparison exercise confirmed the validity of the measurements.

The well established links with other UK and European laboratories along with the formal cooperation agreement greatly assisted the collaboration and communication process between laboratories.

The rapid instigation of an interlaboratory intercomparison exercise provided assurance of the consistency of the analyses to all concerned.

The normal work of the laboratory could not stop instantly. Analyses that were already under way had to be continued until they could be safely put to one side. The laboratory maintained communication with their normal customers. Following discussion, all of our customers were very understanding about accepting delays in their work and expressed satisfaction that they were kept aware of the situation.

As with all incidents involving large numbers of members of the public, there was pressure to produce results with as little delay as possible. Sound management of expectations was essential. It was important to give realistic estimates of both capacity and response times, to allow the incident co-ordinator to plan his response properly.

The pressure to provide results in the shortest possible time meant that the laboratory had to work extended hours including weekends. In the very early phase staff worked long hours but fatigue can rapidly set in. To avoid the possibility of errors work was arranged to limit staff hours.

The HPA carries sufficient stocks for a limited unexpected demand but it is not practicable to hold enough to meet an incident of this magnitude. In this incident in addition to stocks of chemicals and 
laboratory glassware an early problem was availability of urine sample bottles in sufficient quantities. Sampling was initiated on a Friday and the laboratory had only a very limited stock of suitable bottles. All suppliers were extremely helpful in providing material at short notice but if stocks are not held locally there is an inevitable delay that can slow the immediate response.

It was necessary to provide clear written instructions on how to provide 24 hour samples. Many of the people asked to provide samples were not native English speakers and this can cause particular problems.

Transportation of samples from the members of the public to the Chilton laboratory and between collaborating laboratories was needed at short notice. These were biological samples, which caused particular difficulties. The requirement to store the remainder of samples after the removal of the analytical aliquot necessitated the purchase of freezers at very short notice. The disposal of urine samples and bottles also required careful consideration, not because of the radioactive content but because they were biological samples.

Overall the combined resources of many divisions within HPA, not just RPD and LARS, along with the input from the other collaborating laboratories enabled an effective response to be made to an extraordinary event

\section{Acknowledgments}

The authors would like to acknowledge the help and assistance given both by other parts of the HPA and by the collaborating laboratories.

\section{References}

[1] Monitoring programme for polonium-210 carried out by hpa in premises and other sites relevant to public health, 30 November 2006, www.hpa.org.uk.

[2] Leggett R.W. and Eckerman K.F. Sci Total Environ, 275, (2001) 109125.

[3] Spencer H., Holtzman R.B., Kramer l. and IIcewiz F.H., Radiation Research 69 (1977) 166-184.

[4] Naumann M., Hanisc K. and Hartmann M., Rad. Prot. Dos. 79 (1998), 197-200.

[5] Lipsztein J.L., Melo D.R., Sousa W., Dias da Cunha K.M., Azeredo A.M., Julião L. and Santos M. Rad. Prot. Dos., 105 (2003), 1-4.

[6] Al-Arifi, M.N., Alkarfy, K.M., Al-Suwayeh, S.A., Aleissa, K.A., Shabana, E.I., Al-Dhuwaili, A.A. and Al-Hassan, M.I., Journal of Radioanalytical and Nuclear Chem., 269, (2006) 115-118 